\title{
Whole-exome tumor sequencing study in biliary cancer patients with a response to MEK inhibitors
}

\author{
Daniel H. Ahn ${ }^{1}$, Hatice Gulcin Ozer ${ }^{2}$, Baris Hancioglu², Gregory B. Lesinski ${ }^{1}$, Cynthia \\ Timmers ${ }^{1}$ and Tanios Bekaii-Saab ${ }^{1}$ \\ ${ }^{1}$ Department of Internal Medicine, Divison of Medical Oncology, Ohio State University Comprehensive Cancer Center, \\ Columbus, $\mathrm{OH}$, USA \\ 2 Department of Biomedical Informatics, Ohio State University, Columbus, OH, USA \\ Correspondence to: Tanios Bekaii-Saab, email: tanios.saab@osumc.edu
}

Keywords: biliary cancer, whole-exome sequencing, MEK inhibition

Received: August 27, $2015 \quad$ Accepted: December 12, $2015 \quad$ Published: December 16, 2015

\section{ABSTRACT}

We previously conducted a phase-II study with selumetinib (AZD6244), a small molecule inhibitor of MEK1/2, in advanced biliary tract cancers (BTC), where the primary endpoint was response rate. Several patients experienced objective response. These findings were confirmed with MEK162 in a similar patient population. To assess for tumor-specific genetic variants that mediate sensitivity to MEK inhibition in BTC, we performed whole-exome sequencing in patients with an objective response to selumetinib. Normal and tumor DNA from FFPE tissue from two patients who experienced an objective response underwent whole-exome sequencing. Raw sequence reads were processed with GATK workflow and tumor specific variants were identified using MuTect and VarScan2. Ensemble Variant Effect Predictor was used to determine functional consequences of these variants. Copy number changes and potential gene fusion events were also screened. Findings were compared to assess for any commonality between the two tumor samples, and whether the identified variants were intrinsic to the MAPK pathway. 1169 and 628 tumor-specific variants were identified in the two samples. Further analysis demonstrated 60 and 53 functional and novel variants, respectively. Of the identified tumor-specific variants, fusion events or copy number changes, no commonality was seen. Several variants in genes associated with ERK signaling were present in each tumor sample. Although there were no common tumor-specific variants in the two patients who exhibited an objective response to selumetinib, several genes associated with ERK signaling were identified. Confirmatory studies investigating the role of the identified genes and other potential tumor independent factors need further investigation.

\section{INTRODUCTION}

Biliary tract cancers (BTC) comprise of malignancies of the intrahepatic, extrahepatic bile ducts and the gallbladder. The disease is rare, where less than 15,000 cases are diagnosed in the United States each year [1]. Surgical approaches, including resection or liver transplantation are the only curative treatment approaches for BTC [2]. Unfortunately, most patients present with advanced disease at the time of diagnosis. The current standard regimen for untreated advanced BTC is a combination of cytotoxic chemotherapy with gemcitabine and cisplatin, and the disease remains universally fatal, with a median survival that remains less than one year [3]. The poor outcomes with first-line treatment and absence of approved therapies in the refractory setting highlight the need to develop new and more effective treatments for biliary cancers [4-6].

Pre-clinical studies demonstrated genomic alterations in downstream signaling pathway activation and led to an increased interest in investigating novel targeted agents in clinical trials for BTC. Therapies aimed at inhibiting targets in signaling pathways have demonstrated promising pre-clinical activity aimed at $M E K / E R K$, the downstream effector the mitogen activated protein kinase $(M A P K)$ pathway. The initial rationale for 
$M E K$ inhibition was based on the reported high prevalence of activated $R A S$ signaling pathways in biliary cancer [7], with subsequent activation of downstream signaling pathways, including $M A P K$. Additionally, $B R A F$ mutations were shown to be frequently associated with a more sensitive phenotype to $M E K$ inhibition and constitute a survival mechanism for mutant cells [7]. BRAF mutations were identified in up to $22 \%$ of human biliary cancer samples in one study, which were mutually exclusive of KRAS mutations [8], providing further support to $M E K$ inhibition as a rational therapeutic target in BTC.

With the above rationale, we initiated and completed phase II study of selumetinib (AZD6244; AstraZeneca, Manchester, UK), a second-generation, potent, selective and uncompetitive small molecule inhibitor of MAP kinase, MEK1/2, in advanced or metastatic BTC. The primary endpoint of the study was response rate, which was measured according to the Response Evaluation Criteria in Solid Tumors (RECIST) 1.0, assessed by computed tomography (CT) or magnetic resonance imaging (MRI) every 8 weeks [9]. Selumetinib showed preliminary promising activity with a $12 \%$ objective response rate (3 partial response) and a $68 \%$ disease control rate $[10,11]$. Of the three patients who experienced an objective clinical response, 1 patient demonstrated a reduction in tumor marker CA $19-9$ by $60 \%$, while the other two patients were non CA 19-9 secretors. Samples from all 28 patients underwent limited genotyping for $M E K$ relevant targets, $B R A F$ and $K R A S$. We did not identify any $B R A F$ V600E mutations in this patient cohort [11]. Less than $10 \%$ of patients exhibited $K R A S$ mutations that did not correlate with a meaningful response. An expanded analysis that included $K R A S, B R A F, N R A S$, PIK3CA and PTEN in the follow up study with MEK162 in BTC again found no correlation with response [12].

To better understand potential drivers that mediate sensitivity to $M E K$ inhibition in BTC, we conducted a full comprehensive analysis utilizing whole exome sequencing to assess for tumor-specific variants in patients who experienced a response to selumetinib.

\section{RESULTS}

Whole exome sequencing of the patients' tumor and normal sample pairs were conducted. Tumor specific variants, i.e. somatic point mutations, somatic INDELs, and loss of heterozygosity ( $\mathrm{LOH})$ events, were identified. Table 1 lists number of such events and tools used to detect them. We then used Ensembl VEP to determine the effect of the identified variants on genes, transcripts and protein sequence, as well as regulatory regions. We filtered the findings first by identifying all tumor-specific variants, and second, by eliminating any variants found in the dbSNP and ESP databases. We retained all novel variants (those not found in dbSNP or ESP), frame shift and stop gain variants and missense variants with a severe functional consequence (either deleterious by SIFT, or probably/possibly damaging by PolyPhen). As a result, we identified 60 and 53 tumor specific novel and functional variants in sample one and two respectively (Table 2). Figure 1 depicts genome-wide locations of these variants as a PhenoGram [12]. Tumor specific novel and functional variants were analyzed through QIAGEN's Ingenuity ${ }^{\circledR}$ Pathway Analysis (IPA ${ }^{\circledR}$, QIAGEN Redwood City, www.qiagen.com/ingenuity) to assess whether any variants were intrinsic to the RAS/RAF/MAPK pathway (Supplementary Table 3).

We did not identify any commonality in tumorspecific variants (novel and functional) between the two tumor samples or gene variants specific to the RAS/RAF/ MAPK pathway (Supplementary Table 1, Supplementary Table 2). However, several of the identified genes such as NACA, and CUL2 and their associated pathways are linked to $M A P K$ signaling [13-15]. In addition to somatic variants, we examined the two tumor samples displayed for any concordance in gene fusion and copy number events. Acknowledging the limitations of detecting gene fusion events with whole exome sequencing data, we looked for any fusion events of the tumor/normal pairs from our data. Using in house scripts we extracted discordant reads from the alignments and examined potential gene fusion events. We did not detect any fusion events.

Lastly, utilizing VarScan 2, we assessed whether any common changes in copy number were seen between both patient samples. We selected copy number variation (CNV) based on at least $1.5 x$ fold-change in copy number in a given candidate. Copy number changes were found in chromosomes 1, 5, 6, 9, 10, 12, 17, 20, 21 and 22 in one sample, while the second tumor sample had copy number changes in chromosome 11 and 12 (Supplementary Table 3, Supplementary Table 4). We did not identify any similarities in copy number changes and its genes between the two tumor samples.

\section{DISCUSSION}

Biliary tract cancer (BTC) is a rare cancer and is universally lethal, which highlights the urgency to develop novel therapies. The findings from our previous studies consistently suggest that a subset of patients will develop a meaningful objective response to $M E K$ inhibitors [10, 11]. Our initial limited "targeted" analyses of PTEN, KRAS, $B R A F, P I 3 K C A$ and $M E T$ did not elucidate predictors of response to $M E K$ inhibitors with any tumor-specific genetic variants. In order to further assess for tumorspecific genetic variants that mediate sensitivity to $M E K$ inhibition in BTC, we performed whole-exome sequencing in patients with an objective response to single-agent selumetinib.

These additional studies failed to identify any common tumor-specific variants including somatic 
Table 1: Number of somatic variants and LOH events in tumor samples

\begin{tabular}{|l|l|l|l|l|l|l|}
\hline \multirow{2}{*}{ Samples } & \multicolumn{3}{|l|}{ Somatic Point Mutations } & $\begin{array}{l}\text { Somatic } \\
\text { INDEL }\end{array}$ & LOH (Point Mutations) & LOH (INDEL) \\
\cline { 2 - 7 } & MuTect & $\begin{array}{l}\text { MuTect \& } \\
\text { VarScan }\end{array}$ & $\begin{array}{l}\text { VarScan } \\
\text { Somatic }\end{array}$ & VarScan & VarScan & VarScan \\
\hline 1 & 206 & 68 & 72 & 18 & 733 & 72 \\
\hline 2 & 238 & 3 & 81 & 24 & 250 & 32 \\
\hline
\end{tabular}

Table 2: Number of somatic variants in tumor samples and their classification

\begin{tabular}{|l|l|l|}
\hline & Sample 1 & Sample 2 \\
\hline Tumor specific variants & 1169 & 628 \\
\hline Novel variants & 524 & 383 \\
\hline Functional and novel variants & 60 & 53 \\
\hline
\end{tabular}

variants, fusion events and copy number analyses in the examined tumor samples. While the pathway analysis did not demonstrate any of the variants to be genes directly involved with the $M A P K$ pathway, several of the identified functional genomic variants including NACA, and CUL2 and their associated pathways are linked to $M A P K$ signaling [13-15]. N-acetylcysteine amide, the protein encoded by NACA, interacts with $E R K$ signaling and is believed to have an anti-apoptotic effect on cells [16]. CUL2 and its protein product are integrated with hypoxia-inducible factors (HIFs) and are shown to be upregulated by $M A P K$ signaling [17]. Additionally, CUL2 has been identified in other malignancies as a predictor for clinical response to chemotherapy [15]. While limited,

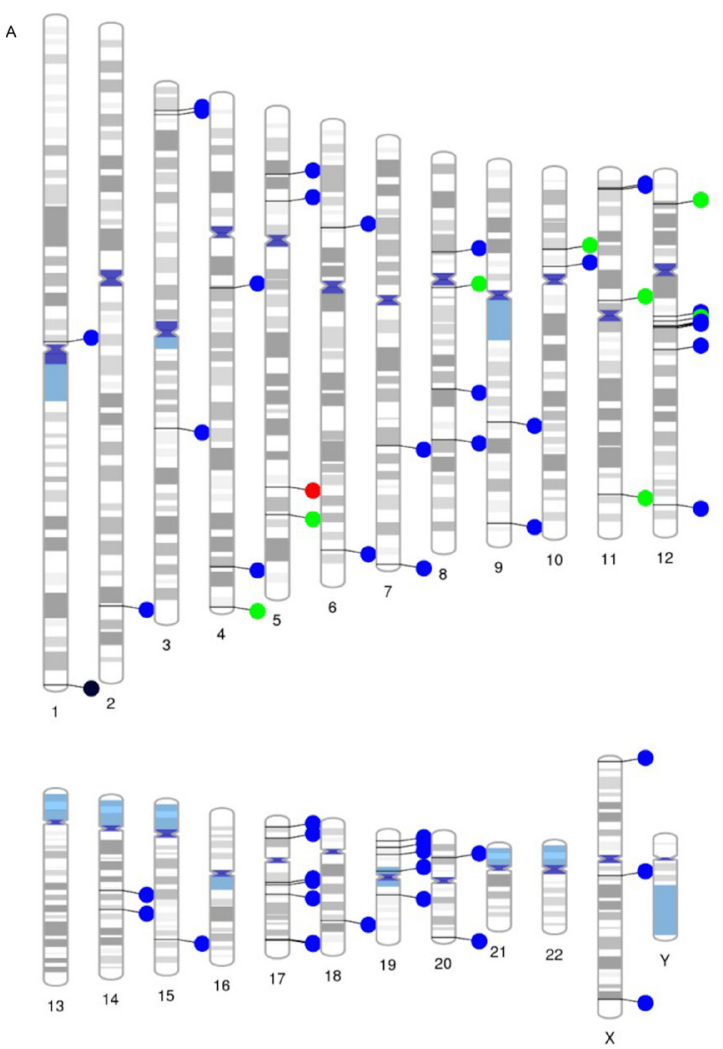

- missense variant $\quad$ splice variant $\quad$ stop gained frameshift variant 

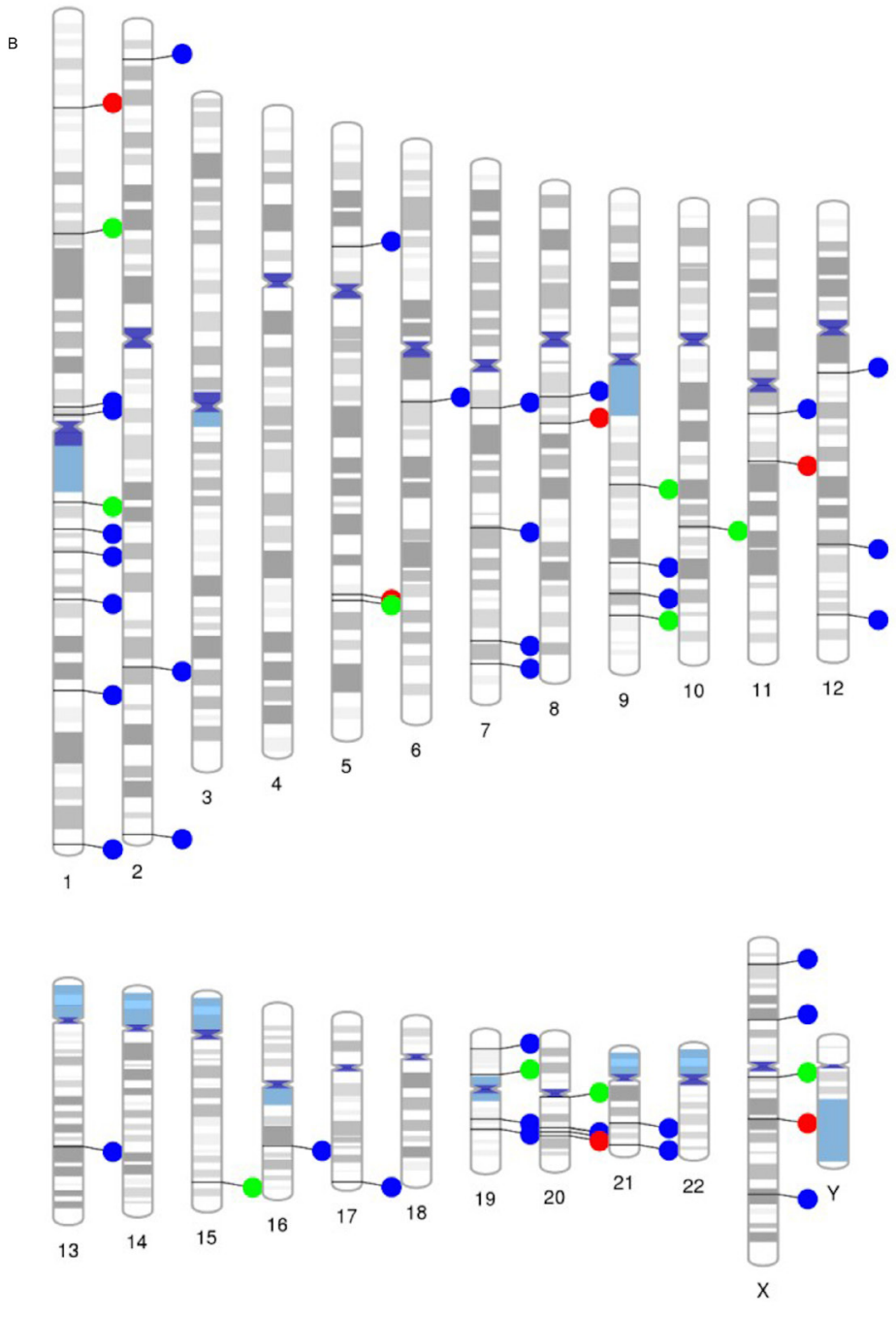

missense variant

splice variant

stop gained

Figure 1: Visualization of novel and functional variants across the genome. Tumor specific variants (missense, splice, stop/ gained and frame shift) are presented with PenoGram for tumor sample one (A) and two (B) respectively. A. Tumor Sample 1. B. Tumor Sample 2.

these interesting findings are novel and worthy of further exploration in prospective studies to assess their potential predictive role for $M E K$ inhibition in BTC.

The absence of definitive genomic variants that may predict for response to $M E K$ inhibition suggest that possible alternative variants or mechanisms may be responsible for the clinical activity consistently observed with MEK inhibitors across 2 studies. It is possible, although unlikely, that expanded whole genome analysis may reveal additional structural variants (such as large insertion/deletions, tandem duplications, gene fusions) that were not identified in this analysis. Alternatively, the oncogenic "driver" may be independent of the tumor itself but rather related to tumor extrinsic factors. Pre-clinical studies suggest a role for activated $M A P K$ pathway as a pro-angiogenic driver and/or as an immunomodulatory effector [18-20]. A phase II randomized study investigating the use of the MEK inhibitor tramenitib vs. a fluoropyrimidine in patients with refractory BTC (SWOG 1310; ClinicalTrials.gov NCT02042443), has incorporated an expanded cytokine and immune cell analysis to confirm the above hypothesis. Finally, the $M A P K$ pathway has been shown to influence epigenetic "drivers" of biliary tract carcinogenesis and future DNA methylation analysis may reveal patterns that would explain the selective clinical response to $M E K$ inhibition in biliary cancer [21- 
23].

The results from our analysis may be limited by the use of DNA derived from FFPE verses fresh frozen samples. However, a next-generation sequencing study of 16 paired fresh-frozen and FFPE tumor samples demonstrated that formalin fixation results in smaller NGS library insert sizes, greater coverage variability and an increase in $\mathrm{C}$ to $\mathrm{T}$ transitions. [24]. In that study, error rate, library complexity and coverage statistics were not significantly different. Comparison of base calls between paired samples showed a high rate of concordance (> 99\%) with $97 \%$ agreement in detected single-nucleotide variants and $>98 \%$ accuracy in collected data in comparison to genotypes from a single-nucleotide polymorphism array platform [24]. These findings along with others demonstrate that the differences between FFPE and fresh tissue are not significant and FFPE samples can be reliably used for whole exome sequencing by NGS [25-27].

Additionally, the use of adjacent normal tissue as germline control is not ideal since we cannot rule out position effect in the data. Moreover, the inclusion of tumor samples from patients that progressed on therapy may have been further informative, however, given the sample size and lack of correlation in the tumor specific variants between the two responders, this may not proved to be useful. To alleviate these limitations, moving forward, we are collecting fresh biopsies when appropriate and whole blood samples for use as a germline control.

In conclusion, our comprehensive analysis through whole-exome sequencing did not identify common tumorspecific changes in patients who responded to $M E K$ inhibition in BTC. Although we did not identify common tumor-specific somatic changes in the two patients who exhibited an objective response to selumetinib, several genes associated with ERK signaling were identified. Confirmatory studies, with the prospective collection of fresh, unfixed tissue and blood samples for germline genetic analysis, investigating the role of the identified genes and other potential tumor independent factors need to be further investigated. An improved understanding of molecular and immunomodulatory abnormalities is key for developing a platform for screening patients for molecularly guided basket clinical trials with available targeted therapies including $M E K, F G F R$ and other inhibitors with promising activity in BTC $[7,28,29]$.

\section{MATERIALS AND METHODS}

The protocol of this study were reviewed and approved by the Ohio State University Institutional Review Board. The methods were carried out in accordance with the approved guidelines and regulations. All the patients provided written informed consent before the initiation of the study. Eligible patients who were included in the initial phase II study were all required to have histologically confirmed advanced biliary tract carcinoma, up to 1 prior systemic anti-cancer therapy, no prior exposure to $M E K$ or $R A F$ inhibitors, and with measurable disease per Response Evaluation Criteria in Solid Tumors (RECIST) [9, 10]. Eligible patients were required to have histologically confirmed biliary tract carcinoma that was surgically unresectable. All patients were required to have either fresh or paraffin-embedded tissue from tumor blocks prior to enrolling onto the study. Radiologic assessment was done by computed tomography or magnetic resonance imaging every 8 weeks, and responses were measured according to RECIST. Three out of twenty-eight patients (12\%) had a confirmed objective response, and 2 of those patients [patient number \#14 and 20] had adequate tissue sample to send for further DNA sequencing [10].

\section{DNA sequencing}

DNA was extracted from surgically resected formalin-fixed paraffin-embedded tissue (FFPE) using the Maxwell 16 FFPE DNA purification kit (Promega, Inc) and quantitated using pico green on a Qubit (Life Technologies). For tumor tissue, an H\&E stained section was marked by a pathologist and tumor containing regions were macro-dissected from serial sections to enrich for tumor content. As a blood sample was not available, germline DNA was derived from FFPE blocks containing adjacent normal tissue. Whole exome capture libraries were constructed from $250 \mathrm{ng}$ of DNA and sequenced by the Broad Institute Genomics Platform on an Illumina HiSeq 2500. The average number of $\mathrm{QC}>30$ reads was 75 million for the normal DNA samples and 250 million reads for the tumor DNA samples with coverage of $68 \%$ and $76 \%$ targeted bases at $>20 \mathrm{X}$ respectively.

\section{Data analysis}

Raw sequence reads were processed and aligned to human reference genome at the Broad Institute using the Genome Analysis Toolkit (GATK) workflow, a software to analyze high-throughput sequencing data, with a primary focus on variant discovery and genotyping, and several measures to ensure data quality assurance [30]. We utilized two well-established tools, MuTect (v1.1.4) and VarScan 2 (v2.3.7), to identify tumor-specific variants for each two patient sample. MuTect is a method developed at the Broad Institute (Cambridge, MA) to identify somatic point mutations in next-generation sequencing (NGS) data of cancer genomes [31]. VarScan2, a platform-independent software tool (developed at Washington University; Saint Louis, Missouri), detects variants (somatic point mutations, insertion/deletions and loss of heterozygosity events) in NGS data [32, 33]. A recent review suggests that MuTect and VarScan 2 detect most variants than any other tools [34]. Therefore, we chose to utilize both tools 
to confirm the findings of each tool and to complement each test given the limitation of MuTect's ability to only detect somatic mutations. Finally, we used Ensembl Variant Effect Predictor (VEP) to annotate and determine functional consequences of tumor specific variants [35]. VarScan 2 was also used to identify copy number changes in tumor samples compared to matching normal samples. Potential gene fusion events were screened using a series of in-house shell and R scripts.

\section{ACKNOWLEDGEMENTS}

None.

\section{CONFLICTS OF INTEREST}

Authors report no relevant conflict of interest.

\section{REFERENCES}

1. Siegel RL, Miller KD and Jemal A. Cancer statistics, 2015. CA: a cancer journal for clinicians. 2015; 65:5-29.

2. Khan SA, Thomas HC, Davidson BR and Taylor-Robinson SD. Cholangiocarcinoma. Lancet. 2005; 366:1303-1314.

3. Valle J, Wasan H, Palmer DH, Cunningham D, Anthoney A, Maraveyas A, Madhusudan S, Iveson T, Hughes S, Pereira SP, Roughton M, Bridgewater J and Investigators ABCT. Cisplatin plus gemcitabine versus gemcitabine for biliary tract cancer. The New England journal of medicine. 2010; 362:1273-1281.

4. Kruth J, Nissen J, Ernst T, Kripp M, Lukan N, Merx K, Hofmann WK, Hochhaus A and Hofheinz RD. Efficacy and safety of capecitabine in combination with docetaxel and mitomycin $\mathrm{C}$ in patients with pre-treated pancreatic, gallbladder, and bile duct carcinoma. Journal of cancer research and clinical oncology. 2010; 136:1845-1851.

5. Lee S, Oh SY, Kim BG, Kwon HC, Kim SH, Rho MH, Kim YH, Rho MS, Jeong JS and Kim HJ. Second-line treatment with a combination of continuous 5-fluorouracil, doxorubicin, and mitomycin-C (conti-FAM) in gemcitabine-pretreated pancreatic and biliary tract cancer. American journal of clinical oncology. 2009; 32:348-352.

6. Oh SY, Jeong CY, Hong SC, Kim TH, Ha CY, Kim HJ, Lee GW, Hwang IG, Jang JS, Kwon HC and Kang JH. Phase II study of second line gemcitabine single chemotherapy for biliary tract cancer patients with 5-fluorouracil refractoriness. Investigational new drugs. 2011; 29:10661072.

7. Tannapfel A, Sommerer F, Benicke M, Katalinic A, Uhlmann D, Witzigmann H, Hauss $\mathrm{J}$ and Wittekind C. Mutations of the BRAF gene in cholangiocarcinoma but not in hepatocellular carcinoma. Gut. 2003; 52:706-712.

8. Rashid A, Ueki T, Gao YT, Houlihan PS, Wallace C, Wang BS, Shen MC, Deng J and Hsing AW. K-ras mutation, p53 overexpression, and microsatellite instability in biliary tract cancers: a population-based study in China. Clinical cancer research. 2002; 8:3156-3163.

9. Therasse P, Arbuck SG, Eisenhauer EA, Wanders J, Kaplan RS, Rubinstein L, Verweij J, Van Glabbeke M, van Oosterom AT, Christian MC and Gwyther SG. New guidelines to evaluate the response to treatment in solid tumors. European Organization for Research and Treatment of Cancer, National Cancer Institute of the United States, National Cancer Institute of Canada. Journal of the National Cancer Institute. 2000; 92:205-216.

10. Bekaii-Saab T, Phelps MA, Li X, Saji M, Goff L, Kauh JS, O’Neil BH, Balsom S, Balint C, Liersemann R, Vasko VV, Bloomston M, Marsh W, Doyle LA, Ellison G, Grever M, et al. Multi-institutional phase II study of selumetinib in patients with metastatic biliary cancers. Journal of clinical oncology. 2011; 29:2357-2363.

11. Finn R, Javle M, Tan B, Weekes C, Bendell J, Patnaik A, Khan G, Laheru D, Anderson L, Christy-Bittel J, Guthrie K, Litwiler K and Bekaii-Saab T. A Phase I Study of MEK Inhibitor MEK162 (ARRY-438162) in patients with biliary tract cancer. J Clin Oncol 30, 2012 (suppl 4; abstr 220).

12. Wolfe D, Dudek S, Ritchie MD and Pendergrass SA. Visualizing genomic information across chromosomes with PhenoGram. BioData mining. 2013; 6:18.

13. Arai $\mathrm{K}$, Lee SR, van Leyen $\mathrm{K}$, Kurose $\mathrm{H}$ and Lo EH. Involvement of ERK MAP kinase in endoplasmic reticulum stress in SH-SY5Y human neuroblastoma cells. Journal of neurochemistry. 2004; 89:232-239.

14. Hotokezaka Y, van Leyen K, Lo EH, Beatrix B, Katayama I, Jin $\mathrm{G}$ and Nakamura T. alphaNAC depletion as an initiator of ER stress-induced apoptosis in hypoxia. Cell death and differentiation. 2009; 16:1505-1514.

15. Metzger R, Heukamp L, Drebber U, Bollschweiler E, Zander T, Hoelscher AH and Warnecke-Eberz U. CUL2 and STK11 as novel response-predictive genes for neoadjuvant radiochemotherapy in esophageal cancer. Pharmacogenomics. 2010; 11:1105-1113.

16. Gong X, Celsi G, Carlsson K, Norgren S and Chen M. $\mathrm{N}$-acetylcysteine amide protects renal proximal tubular epithelial cells against iohexol-induced apoptosis by blocking p38 MAPK and iNOS signaling. American journal of nephrology. 2010; 31:178-188.

17. Sang N, Stiehl DP, Bohensky J, Leshchinsky I, Srinivas $\mathrm{V}$ and Caro J. MAPK signaling up-regulates the activity of hypoxia-inducible factors by its effects on p300. The Journal of biological chemistry. 2003; 278:14013-14019.

18. Hood JD, Frausto R, Kiosses WB, Schwartz MA and Cheresh DA. Differential alphav integrin-mediated RasERK signaling during two pathways of angiogenesis. The Journal of cell biology. 2003; 162:933-943.

19. Dormond O, Madsen JC and Briscoe DM. The effects of mTOR-Akt interactions on anti-apoptotic signaling in vascular endothelial cells. The Journal of biological 
chemistry. 2007; 282:23679-23686.

20. Maemura K, Natsugoe S and Takao S. Molecular mechanism of cholangiocarcinoma carcinogenesis. Journal of hepato-biliary-pancreatic sciences. 2014; 21:754-760.

21. Letelier P, Brebi P, Tapia O and Roa JC. DNA promoter methylation as a diagnostic and therapeutic biomarker in gallbladder cancer. Clinical epigenetics. 2012; 4:11.

22. Andresen K, Boberg KM, Vedeld HM, Honne H, Jebsen P, Hektoen M, Wadsworth CA, Clausen OP, Lundin KE, Paulsen V, Foss A, Mathisen O, Aabakken L, Schrumpf E, Lothe RA and Lind GE. Four DNA methylation biomarkers in biliary brush samples accurately identify the presence of cholangiocarcinoma. Hepatology. 2015; 61:1651-1659.

23. Chiang NJ, Shan YS, Hung WC and Chen LT. Epigenetic regulation in the carcinogenesis of cholangiocarcinoma. The international journal of biochemistry \& cell biology. 2015.

24. Spencer DH, Sehn JK, Abel HJ, Watson MA, Pfeifer JD and Duncavage EJ. Comparison of clinical targeted next-generation sequence data from formalin-fixed and fresh-frozen tissue specimens. The Journal of molecular diagnostics. 2013; 15:623-633.

25. Van Allen EM, Wagle N, Stojanov P, Perrin DL, Cibulskis K, Marlow S, Jane-Valbuena J, Friedrich DC, Kryukov G, Carter SL, McKenna A, Sivachenko A, Rosenberg M, Kiezun A, Voet D, Lawrence M, et al. Whole-exome sequencing and clinical interpretation of formalin-fixed, paraffin-embedded tumor samples to guide precision cancer medicine. Nature medicine. 2014; 20:682-688.

26. Carrick DM, Mehaffey MG, Sachs MC, Altekruse S, Camalier C, Chuaqui R, Cozen W, Das B, Hernandez BY, Lih CJ, Lynch CF, Makhlouf H, McGregor P, McShane LM, Phillips Rohan J, Walsh WD, et al. Robustness of Next Generation Sequencing on Older Formalin-Fixed ParaffinEmbedded Tissue. PloS one. 2015; 10:e0127353.

27. Munchel S, Hoang Y, Zhao Y, Cottrell J, Klotzle B, Godwin AK, Koestler D, Beyerlein P, Fan JB, Bibikova $\mathrm{M}$ and Chien J. Targeted or whole genome sequencing of formalin fixed tissue samples: potential applications in cancer genomics. Oncotarget. 2015; 6:25943-25961. doi: 10.18632/oncotarget.4671.

28. Borad MJ, Champion MD, Egan JB, Liang WS, Fonseca R, Bryce AH, McCullough AE, Barrett MT, Hunt K, Patel MD, Young SW, Collins JM, Silva AC, Condjella RM, Block M, McWilliams RR, et al. Integrated genomic characterization reveals novel, therapeutically relevant drug targets in FGFR and EGFR pathways in sporadic intrahepatic cholangiocarcinoma. PLoS genetics. 2014; 10:e1004135.

29. Sia D, Losic B, Moeini A, Cabellos L, Hao K, Revill K, Bonal D, Miltiadous O, Zhang Z, Hoshida Y, Cornella H, Castillo-Martin M, Pinyol R, Kasai Y, Roayaie S, Thung SN, et al. Massive parallel sequencing uncovers actionable FGFR2-PPHLN1 fusion and ARAF mutations in intrahepatic cholangiocarcinoma. Nature communications.
$2015 ; 6: 6087$

30. Van der Auwera GA, Carneiro MO, Hartl C, Poplin R, Del Angel G, Levy-Moonshine A, Jordan T, Shakir K, Roazen D, Thibault J, Banks E, Garimella KV, Altshuler D, Gabriel $\mathrm{S}$ and DePristo MA. From FastQ data to high confidence variant calls: the Genome Analysis Toolkit best practices pipeline. Current protocols in bioinformatics / editoral board, Andreas D Baxevanis [et al]. 2013; 11:11 10 11-11 1033 .

31. Cibulskis K, Lawrence MS, Carter SL, Sivachenko A, Jaffe D, Sougnez C, Gabriel S, Meyerson M, Lander ES and Getz G. Sensitive detection of somatic point mutations in impure and heterogeneous cancer samples. Nature biotechnology. 2013; 31:213-219.

32. Koboldt DC, Zhang Q, Larson DE, Shen D, McLellan MD, Lin L, Miller CA, Mardis ER, Ding L and Wilson RK. VarScan 2: somatic mutation and copy number alteration discovery in cancer by exome sequencing. Genome research. 2012; 22:568-576.

33. Koboldt DC, Chen K, Wylie T, Larson DE, McLellan MD, Mardis ER, Weinstock GM, Wilson RK and Ding L. VarScan: variant detection in massively parallel sequencing of individual and pooled samples. Bioinformatics. 2009; 25:2283-2285.

34. Wang Q, Jia P, Li F, Chen H, Ji H, Hucks D, Dahlman KB, Pao $\mathrm{W}$ and Zhao Z. Detecting somatic point mutations in cancer genome sequencing data: a comparison of mutation callers. Genome medicine. 2013; 5:91.

35. McLaren W, Pritchard B, Rios D, Chen Y, Flicek P and Cunningham F. Deriving the consequences of genomic variants with the Ensembl API and SNP Effect Predictor. Bioinformatics. 2010; 26:2069-2070. 